\title{
ERRATA
}

\section{Asymptotic profiles of a decaying contaminant in transport through a porous medium}

\author{
M. ESCOBEDO and R. E. GRUNDY
}

Vol. 7 (1996), pp. 395-416

The publisher apologises for an oversight which resulted in an error in the above paper.

The following three figures were inadvertently published in an incomplete form, with some of the labelling omitted.

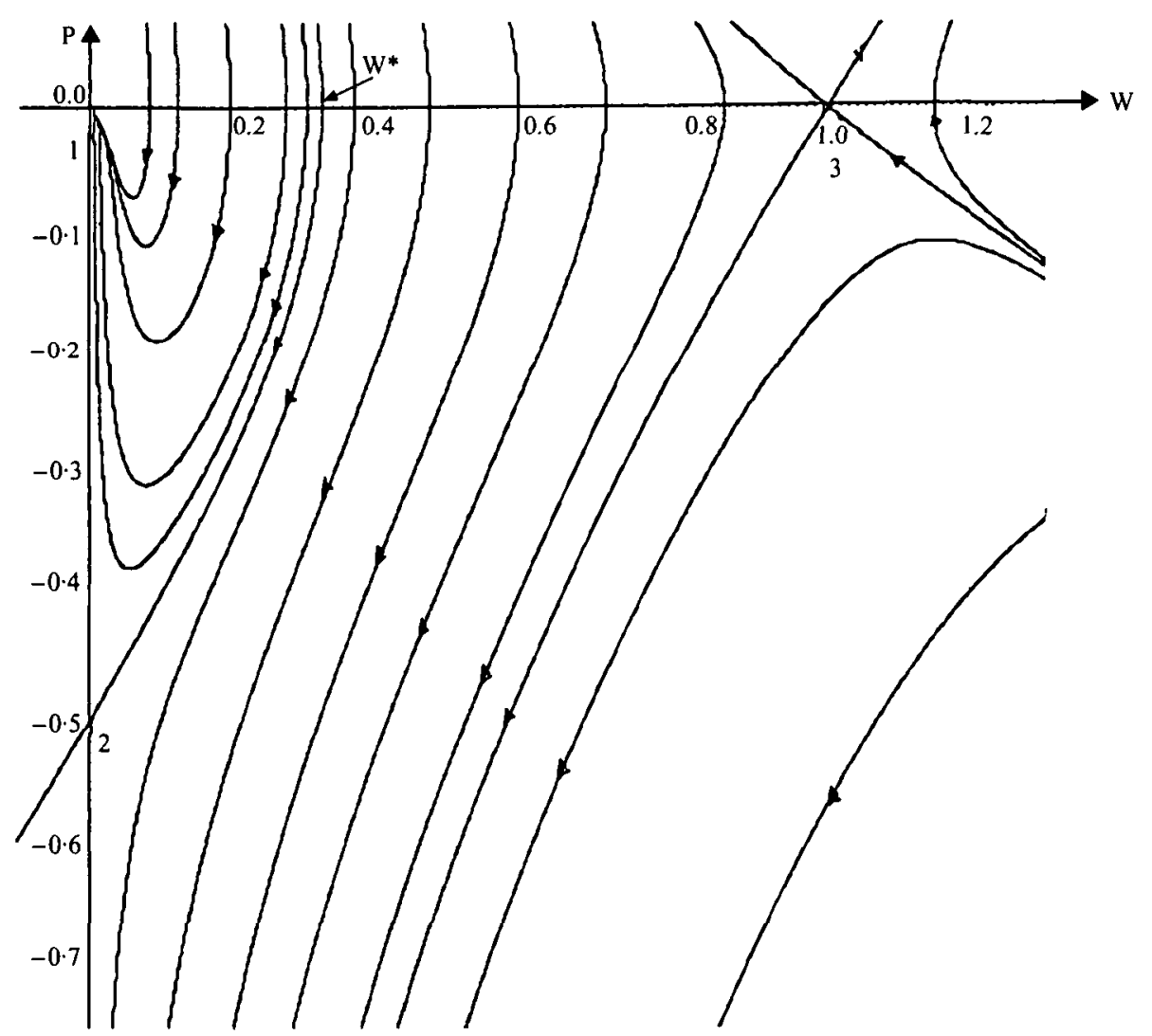

Figure 1. Computed integral curves for a typical case when $0<\alpha<\alpha_{2}$. Here $m=2, \gamma=1, \alpha=1$ and $\alpha_{2}=1.618$. Arrows indicate directions of increasing $\zeta$. 


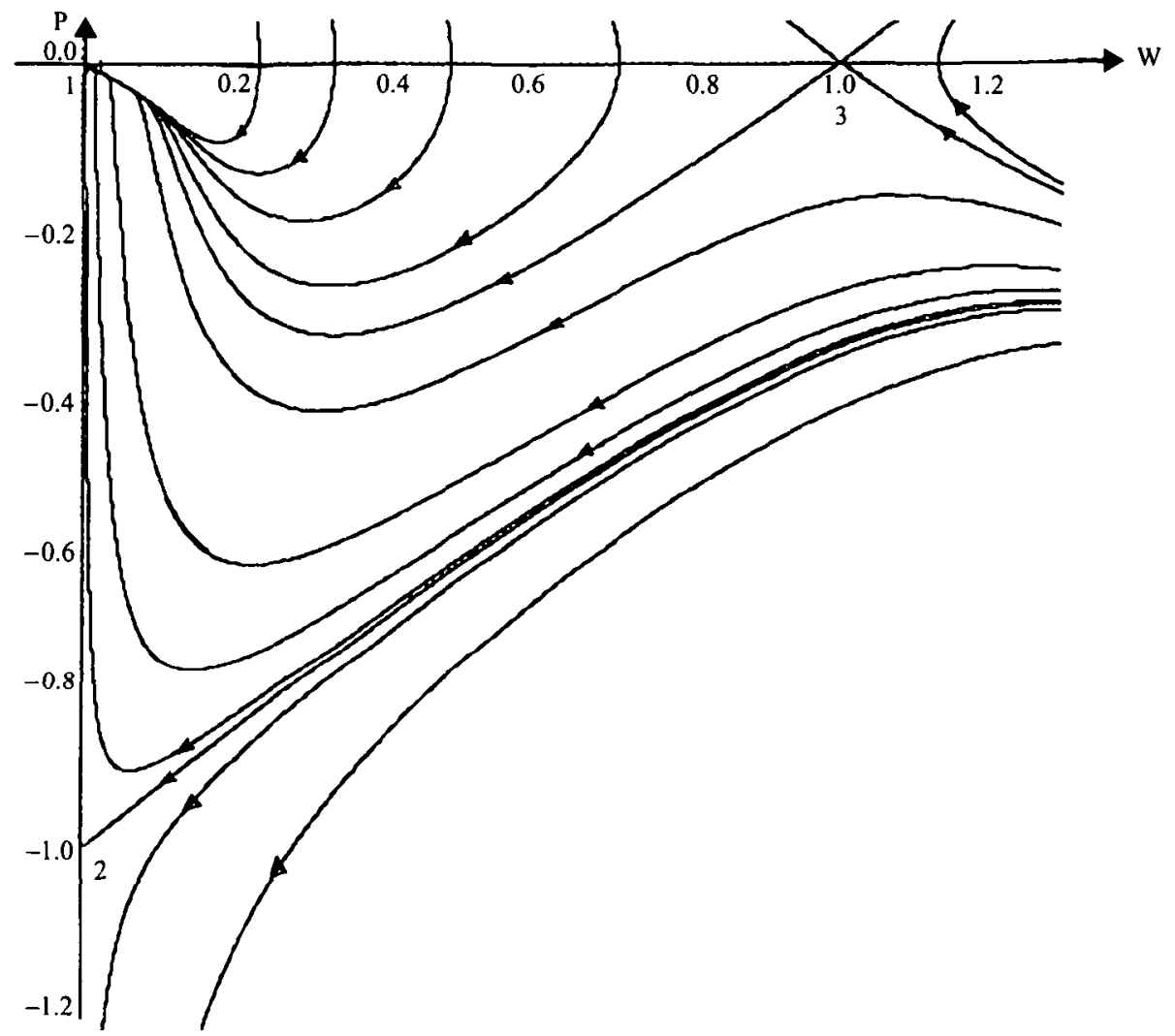

FIGURE 2. Computed integral curves for a typical case when $\alpha>\alpha_{2}$. Here $m=2.0, \gamma=1.0$, $\alpha=2.0$ and $\alpha_{2}=1.618$. Arrows indicate directions of increasing $\zeta$.

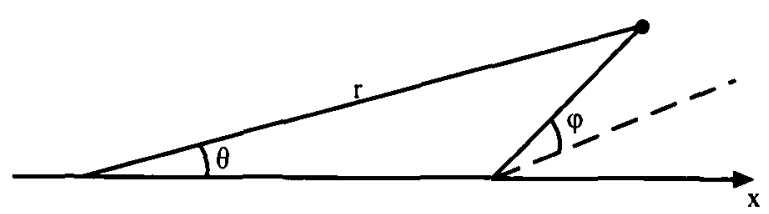

FIGURE 3. Coordinate system in three dimensions. 